\title{
Interfaces
}

\section{Tressage de sensation et hypersensibilité dans la poésie keatsienne}

\section{Laure-Hélène Anthony-Gerroldt}

\section{(2) OpenEdition}

1 Journals

\section{Édition électronique}

URL : http://journals.openedition.org/interfaces/241

DOI : 10.4000/interfaces.241

ISSN : 2647-6754

Éditeur :

Université de Bourgogne, Université de Paris, College of the Holy Cross

\section{Édition imprimée}

Date de publication : 1 janvier 2015

Pagination : 233-249

ISBN : 9780983175247

ISSN : 1164-6225

\section{Référence électronique}

Laure-Hélène Anthony-Gerroldt, «Tressage de sensation et hypersensibilité dans la poésie keatsienne », Interfaces [En ligne], 36 | 2015, mis en ligne le 01 janvier 2018, consulté le 07 janvier 2021. URL : http://journals.openedition.org/interfaces/241 ; DOI : https://doi.org/10.4000/interfaces. 241

Les contenus de la revue Interfaces sont mis à disposition selon les termes de la Licence Creative Commons Attribution 4.0 International. 


\section{TRESSAGE DE SENSATION \\ ET HYPERSENSIBILITE DANS LA POESIE KEATSIENNE}

Laure-Hélène Anthony-Gerroldt

La synesthésie, phénomène neurologique aujourd'hui reconnu, suscite depuis plusieurs années un regain d'intérêt dans le monde scientifique comme le confirme la parution, en 2011, de l'article d'Hervé-Pierre Lambert « La synesthésie. Vues de l'intérieur », dans lequel l'auteur répertorie les évolutions historiques de l'étude du concept. En neuropsychologie, la synesthésie est un trouble de la perception sensorielle qui produit des associations prévisibles, mais involontaires, entre différents modes de perception. Ainsi, la stimulation d'un sens donné activera simultanément le sens stimulé et un ou plusieurs des autres organes sensoriels. Partant de cette définition, Reuven Tsur, qui s'est intéressé aux rapports entre le phénomène neurologique et le procédé littéraire dans son article « Issues in Literary Synaesthesia », explique que la synesthésie constitue une exception dans une réalité de sens différenciés (Tsur, 30).

En littérature, il s'agit en revanche de la juxtaposition de termes issus des champs lexicaux applicables aux cinq sens. Les mots choisis appartiennent à des domaines sensoriels distincts, mais ne font pas référence à une réalité de sens différenciés. La synesthésie permet alors de décrire un monde hyperesthésique (Tsur, 30).

L'étymologie du nom corrobore cette idée : la synesthésie au sens large décrit des aptitudes perceptives, affectives et intellectuelles. Le grec ancien décline le terme sous trois formes grammaticales. Le verbe « synaisthanomaï » décrit la faculté de ressentir et de partager les sentiments de l'autre ; le nom « synaisthêsis » désigne la perception simultanée de sentiments, de sensations ou d'expériences de natures et d'origines diverses ; le participe «synaisthêtikos » s'applique enfin à une personne ayant conscience de quelque chose ou d'elle-même. Les fonctions de la synesthésie semblent donc bien dépasser celles des cinq sens ordinaires : faisant disparaître les barrières entre les sens, elle permet également le partage des émotions et pose la question de la conscience. C'est ce concept que Keats décline dans son œuvre poétique.

Il existe, par ailleurs, une seconde définition littéraire de la synesthésie. Le concept peut en effet se manifester par le transfert des qualités d'un sens vers un autre afin d'atteindre ou d'exprimer un état de plénitude sensorielle. Selon Erika von Erhardt-Siebold, cette quête perpétuelle est le propre 
de Shelley qui cherchait à découvrir une tonalité venue d'un autre monde, unissant en un même sens la musique, la lumière et les sensations physiques (Erhardt-Siebold, 590). ${ }^{1}$

Cette fusion des perceptions permettrait alors au poète d'atteindre un monde super-sensible représentatif de la véritable union entre âme et corps. Cependant, si Shelley utilise la synesthésie, c'est parce qu'il tente de donner de la substance à des sensations indescriptibles (Erhardt-Siebold, 590-91). L'expérience sensorielle du poète n'est pas tangible ; il doit par conséquent y transférer les attributs des sens dont le degré d'ancrage corporel est le plus élevé - l'odorat, le goût et le toucher - pour pouvoir la décrire et la rendre accessible à son lecteur. Elle se rapproche en cela des états d'indolence et de demiconscience qui traverse l'œuvre de Keats. ${ }^{2}$

Cependant, Keats n'emploie pas la synesthésie de la même manière que Shelley ou ses autres contemporains. Son œuvre repose sur le caractère concret et la richesse de l'expérience sensorielle, qu'il décrit et qui est transcrite dans le texte poétique et épistolaire sous des formes variées transcendant les catégories traditionnellement dévolues aux cinq sens.

Il s'agit donc de se demander comment ces catégories sont dépassées et quelles formes nouvelles sont alors créées. Je vais explorer les particularités de l'expérience sensorielle chez Keats, où, comme on va le voir, les sens ne sont pas soumis à une hiérarchie stricte. La synesthésie devient alors un sens à part, davantage issu du tressage des perceptions que de leur confusion, de leur juxtaposition ou du transfert de leurs qualités. Elle se présente ainsi comme une manifestation littéraire du désir de complétude caractéristique de l'œuvre keatsienne.

Le concept de hiérarchie sensorielle établit un schéma selon lequel la vue et l'ouïe priment sur l'odorat, le goût et le toucher. Selon Naomi Segal, le motif est ancré dans la grammaire des langues qui reflète une hiérarchie allant des sens supérieurs aux sens inférieurs. En effet, il existe en anglais, comme en français, trois formes verbales primaires associées à la vue et à l'ouïe contre une seule pour le toucher, le goût et l'odorat. Ainsi, on peut avoir l'air fatigué (« look tired »), regarder (« look »/ « watch ») et voir ( « see »). De la même façon, on peut sembler intéressé (« sound interested »), écouter $(«$ listen $)$ ) et entendre (« hear »). Ces formes verbales correspondent à trois modes de perception, que

1 Quoique l'article soit daté, il est le seul qui, à ma connaissance, compare directement l'utilisation de la synesthésie chez quatre des grands poètes romantiques anglais.

2 La brièveté de l'article, qui se concentre sur la singularité formelle de la synesthésie keatsienne, ne permet malheureusement pas de développer sur les états de réceptivité exacerbée ou d'indolence qui entretiennent, par ailleurs, une relation étroite à la synesthésie et la sensation dans l'œuvre du poète. 
Segal nomme respectivement « passive », « active-focused » et « active-unfocused ». En revanche, les trois autres sens, qualifiés d'inférieurs, ont un vocabulaire beaucoup moins différencié qui se limite, en anglais, aux verbes « smell », " touch » et « taste ». ${ }^{3}$ On utilise le même verbe quel que soit le mode perception. Reprenant un exemple choisi par Segal, on dira ainsi : « I smell a rose; I smell burning; I smell funny ». Cette indifférenciation lexicale permet à l'auteur de conclure que les sens inférieurs apparaissent moins définis et contribuent donc moins souvent aux métaphores liées à la compréhension ou à la connaissance.

Denise Gigante rappelle par ailleurs que les Grecs appliquaient déjà le concept de hiérarchie sensorielle aux cinq sens humains reconnus :

In philosophical discourse since Plato, $[\ldots]$, « the most basic distinction that separates sight and hearing from smell, taste, and touch concerns the apparent degree of involvement of the body in the operations of the senses $» .[\ldots]$ Whereas sight and hearing were traditionally considered to be oriented toward the external world, the lower, bodily senses were thought to say more about the perceiving subject than the object of taste. (Gigante, 496)

La distinction la plus apparente entre les sens naît de leur degré d'ancrage corporel. Contrairement aux trois autres sens qui ne peuvent être activés que grâce à un contact direct, ou semi direct dans le cas de l'odorat, avec le corps, la vue et l'ouïe ne nécessitent aucun contact physique pour être stimulées. Dès lors, elles sont considérées comme des sens nobles en relation étroite avec l'âme et plus à même de provoquer le détachement nécessaire à l'élévation de la sensibilité humaine vers des hauteurs philosophiques ou spirituelles. Le goût, l'odorat et le toucher font quant à eux référence à des réalités plus concrètes.

Appliquée à la synesthésie, la hiérarchie sensorielle permet donc de mettre en relation une expérience tangible, comme le fait d'avoir chaud, avec une impression sensorielle plus lointaine, plus diffuse, comme la lumière ou le son. Yeshayahu Shen et Michal Cohen, qui ont répertorié et analysé les mécanismes de nombreuses métaphores synesthésiques chez les Romantiques anglais, expliquent du reste que les transferts se font majoritairement depuis les sens inférieurs vers les sens supérieurs justement parce que les sens dits inférieurs sont plus concrets et donc plus accessibles sur le plan cognitif. Ainsi, ils peuvent servir d'extensions sémantiques permettant de définir et de comprendre

Segal, Naomi. «'L'échange de deux fantaisies et le contact de deux épidermes' : Skin and Desire. » Sensual Reading: New Approaches to Reading in its Relations of the Senses. Ed. Syrotinski, Michael, and Maclachlan, Ian. London: Associated University Presses, 2001. 17-38. 
les sensations abstraites liées à la vue et à l'ouïe (Shen-Cohen, 112). Par conséquent, la synesthésie favorise les interactions entre le corps et l'esprit, ou le réel et l'imaginaire, la vie et la création. On retrouve ici la visée des transferts sensoriels de Shelley, mais également de Wordsworth qui écrit dans son très célèbre poème « Tintern Abbey » : «While with an eye made quiet by the power / Of harmony, and the deep power of joy, / We see into the life of things » (vers 47-49). ${ }^{4}$ Grâce à l'influence positive de l'ouïe sur la vue, il découvre la vie intime des choses.

Keats propose une utilisation différente des sensations que ses contemporains car son expérience sensorielle ne repose plus sur la hiérarchie traditionnellement établie. Dans son œuvre poétique et épistolaire, la relation des sens à l'imagination est étendue aux domaines de l'ouïe et des touchers. Elle dépasse également les domaines sensoriels traditionnels en faisant de la respiration un sens à valeur synesthésique.

Comme chez la plupart des poètes, la vue entretient chez Keats une relation étroite avec l'imagination et la création. Leon Waldoff rappelle dans la préface à Keats and the Silent Work of Imagination que cette association nous vient du latin « imaginatio », qui désigne la faculté de former des images dans l'esprit (Waldoff, 7). Plus encore, elle est contenue dans la locution anglo-saxonne qui sert de périphrase pour l'imagination : " the mind's eye ». Apparue pour la première fois chez Chaucer dans The Man of Law's Tale, aux environs de 1390, l'expression sera consacrée par Shakespeare qui l'utilisera sous sa forme actuelle dans Hamlet. En anglais comme en latin, imaginer, c'est donc voir de l'intérieur. Ce rapport est illustré à plusieurs reprises dans les écrits de Keats, pour qui l'imagination a une fonction visuelle propre, intimement liée à la création.

Or, si la vue est la capacité de percevoir le monde qui nous entoure, la vision poétique constitue en revanche ce que seul le poète peut voir et rendre apparent dans son art. Cette distinction est clairement formulée dans la lettre-journal du 17 au 27 septembre 1819 adressée à George et Georgiana Keats : "You speak of Lord Byron and me - There is this great difference between us. He describes what he sees - I describe what I imagine - Mine is the hardest task $\gg .^{5}$ La vision ne fait qu'une avec l'imagination du poète ; elle est l'œil de son esprit par lequel il peut percevoir un monde poétique caché.

4 Wordsworth, William. « Tintern Abbey ». William Wordsworth: The Major Works (including The Prelude). Ed. Stephen Gill. Oxford: Oxford University Press (Oxford World's Classics), 2008.

5 Keats, John. John Keats: Selected Letters, éd. Robert Gittings, revised by Jon Mee. Oxford: Oxford University Press. 2002. To George and Georgiana Keats, 17-27 September, 1819, pp. 283-307. 
On retrouve également cette idée dans les poèmes. Ainsi, dans « I stood tip-toe upon a little hill », l'observation de la nature déclenche les visions qui deviennent poésie : " And many pleasures to my vision started; / So I straightway began to pluck a posey / Of luxuries bright, milky, soft, and rosy » (vers 26-28). Dans les vers suivants, le locuteur du poème décrit un nouveau paysage, une vision poétique qui est le produit de son imagination.

Néanmoins, ce qui fait la particularité de l'œuvre de Keats est que le rapport privilégié entre imagination et perception que reflète la locution « the mind's eye » est étendu aux autres sens. Ainsi, Keats met en relation l'ouïe et la capacité créative du poète lorsqu'il emploie l'expression « my fancy's ear » dans le poème « Unfelt, unheard, unseen ». Il existe donc pour lui une forme d'ouïe qui appartient au domaine de l'imagination. Pour mieux saisir cette idée, on peut se tourner vers le sonnet « How many bards guild the lapses of time! » dans lequel Keats écrit des poètes des temps passés :

And often when I sit me down to rhyme

These will in throngs before my mind intrude:

But no confusion, no disturbance rude

Do they occasion; 'tis a pleasing chime (vers 5-8).

Le poète qui s'apprête à composer est interrompu par les bardes des temps anciens qui viennent murmurer à son oreille. En influençant sa perception de la nature, ils deviennent une source d'inspiration pour lui : les vers suivants énumèrent les bruits environnants, depuis le chant des oiseaux jusqu'au roulement des vagues, qui se transforment en musique. La mélodie vient envelopper l'oreille du poète qui peut alors se mettre à l'œuvre et créer.

De la même façon, quoiqu'il puisse paraître étonnant d'associer le goût, le toucher et l'odorat au processus de création poétique, l'importance que Keats accorde à ces sens rend le rapprochement moins insolite.

Il convient tout d'abord de préciser que si l'odorat, le toucher et le goût ont chacun une importance individuelle, ils peuvent toutefois être mis en relation et étudiés ensemble. En effet, tous coïncident avec une forme particulière de toucher : le goût peut se concevoir comme une forme de toucher palatal et l'odorat, de toucher ou contact, nasal. Ce que l'on qualifie habituellement de « toucher » représente en réalité le toucher épidermique, qui regroupe les perceptions et sensations externes, et le toucher épithélial et muqueux, qui correspond aux perceptions et sensations internes.

C'est d'ailleurs cette qualité de toucher épithélial ou muqueux qui permet de rapprocher les trois formes de toucher. L'odorat engage ainsi l'action des tissus du nez tandis que le goût fait travailler 
ceux de la langue, du palais et des muqueuses des joues dans la bouche. Keats avait déjà remarqué la relation étroite entre ce que je qualifie de " formes de toucher ». En effet, comme le rappelle Denise Gigante, au printemps 1816, alors qu'il était étudiant à Guy's Hospital, Keats notait dans son carnet d'anatomie et de physiologie, en citant ses professeurs :

The different sensations reside in peculiar structures as the toes \& fingers which have papillae through which the sense of feeling - the papillae of the Tongue are different from those of the Toes \& fingers \& are larger - the papillae of the Membrane of the nose are very minute \& sensitive. (Gigante, 496)

Il apparaît dans ces quelques lignes que Keats met le toucher, le goût et l'odorat en relation directe à travers l'emploi du terme « papillae ». Le jeune homme rapproche le fonctionnement des trois organes sensoriels sans gommer les nuances qui existent entre eux.

Par suite, lorsque Keats écrit « the palate of my mind » au vers 13 de son sonnet « To Fanny », il emploie une tournure très proche de « my mind's eye ». On comprend donc que le poète nous dévoile l'existence d'une imagination par le goût, par l'odorat et par le toucher. Cette révélation est confirmée par l'expression « touch has a memory » utilisée au vers 4 de « Lines to Fanny ». En effet, le processus de création poétique, qui dépend de l'imagination, repose également sur le travail de la mémoire et des sensations. Par l'association de sensations et de perceptions récentes à des souvenirs et des émotions, on crée de nouvelles images qui prennent ici la forme de poèmes.

Ainsi, le locuteur de « Lines to Fanny », après avoir annoncé que le toucher avait une mémoire, décrit l'effet produit par la poitrine, le souffle chaud, les lèvres de Fanny, et c'est bien le souvenir de ces moments qui engendre l'écriture du poème. La création poétique est exaltée par les perceptions et les sensations tactiles. Par conséquent, la hiérarchie traditionnelle des sens ne s'applique pas à la poésie de Keats, dans la mesure où la création et l'imagination sont stimulées par tous les sens humains sans distinction. Pour Keats, tout sens doit permettre d'accéder à l'imagination. Aussi, bien que le poète ait moins souvent recours à l'odorat qu'aux autres sens, il se tourne vers cette modalité perceptive lorsque la vue lui fait défaut dans « Ode to a Nightingale ». L'imagination olfactive l'aide à envisager, comprendre et créer son monde et son poème. Toutefois, si la hiérarchie traditionnelle ne s'applique pas à Keats, le poète utilise néanmoins les sens liés au toucher et au contact plus spontanément. ${ }^{6}$ On

6 Une étude complète des images sensorielles permettrait d'en répertorier le nombre et la nature pour étudier la fréquence d'utilisation de chaque sens. Voir également : Ullmann, Stephen de. « Romanticism and Synaesthesia: a Comparative Study of Sense Transfer in Keats and Byron ». PMLA: Publications of the Modern Language Association of America 60.3 (1945): 811-827. Bien qu'il s'appuie sur la définition de synesthésie comme transfert, l'article de Stephen de Ullman propose une étude chiffrée du procédé chez Keats et Byron. 
peut relier cette préférence personnelle à l'anti-intellectualisme du jeune homme, pour qui les images les plus frappantes ne passent pas forcément par la vue.

Par ailleurs, la remise en cause de la hiérarchie ne tient pas uniquement à la relation entre sensation et création établie jusqu'ici. En effet, la respiration apparaît comme un sens à part entière dans l'œuvre keatsienne, ce qui enrichit l'expérience sensorielle d'un sixième sens et accentue le caractère obsolète de la hiérarchie traditionnelle. Les références à l'air et au souffle, très présentes dans les écrits du jeune homme, montrent bien que le poète perçoit et comprend le monde qui l'entoure grâce à la respiration.

Le sonnet «After dark vapours have oppressed our plains » en est un bon exemple qu'il est pertinent de citer. En effet, bien que Keats n'évoque que rarement les problèmes respiratoires humains que les études médicales et la maladie lui ont rendus familiers, il les fait apparaître sous forme de vapeurs nauséabondes :

After dark vapours have oppressed our plains

For a long dreary season, comes a day

Born of the gentle South, and clears away

From the sick heavens all unseemingly stains.

$[\ldots]$

And calmest thoughts come round us - as of leaves

Budding - fruit ripening in stillness - autumn suns

Smiling at eve upon the quiet sheaves -

Sweet Sappho's cheek - a sleeping infant's breath - (vers 1- 12).

Le sonnet évoque le passage de l'hiver au printemps. Toutefois, plutôt que d'insister sur le bourgeonnement des feuilles et l'arrivée du soleil, Keats choisit de se concentrer sur les changements éoliens liés au passage des saisons. L'hiver prend la forme d'effluves oppressants qui empêchent la respiration et créent un sentiment d'angoisse. Ces vapeurs ont rendu les cieux, et par métaphore les populations, malades. Le printemps, représenté par le vent doux du sud, arrive alors pour chasser la maladie et rétablir, par son souffle sain, la santé de la nature et des hommes. C'est pourquoi, parmi les images et pensées qu'apporte ce vent bienfaiteur, on retrouve la respiration douce d'un enfant, qui représente également le renouveau et la santé.

L'air et le souffle constituent donc pour Keats une preuve de vie et de santé. Dans l'œuvre keatsienne, tout est souffle et respiration : les boudoirs végétaux d'Endymion sont pleins de respirations apaisées et apaisantes - « quiet breathing » (vers 5). Les locuteurs des poèmes dont l'objet est une 
femme aimée prêtent une attention particulière à la respiration de leur amante. Le locuteur du sonnet «Bright Star» demande ainsi à entendre pour toujours la respiration pleine de tendresse de son amante et écrit : « Still, still to hear her tender-taken breath » (vers 13). L'amant doit veiller à la respiration de la femme aimée comme si sa propre vie en dépendait. Lorsque l'être aimé ne respire plus et cesse d'être, le poète-amant ne semble plus pouvoir continuer de vivre. C'est pourquoi la respiration de Lamia dans le poème éponyme devient le souffle de mort ou « death-breath » (vers 299) au moment où elle expire et emporte avec elle son bien-aimé Lycius.

De plus, la respiration est par essence liée aux autres sens et aux émotions : entendue, ressentie aussi bien à l'intérieur qu'à l'extérieur du corps, elle peut également se voir ou révéler l'apaisement, la douleur et l'angoisse. On peut donc bien parler de sens respiratoire à valeur synesthésique : on perçoit et l'on ressent le souffle par l'action conjointe de tous les sens.

La relation entre sensation et création poétique, qui fait la singularité des sens keatsiens, est également évidente pour la respiration car contenue dans le terme polysémique, en anglais comme en français, d'inspiration. En poésie, elle prend même une forme écrite. Chez Keats, l'emploi du tiret marque en effet les plus hauts moments d'inspiration poétique ainsi que l'influence du souffle sur l'écriture. ${ }^{7}$ Aussi, lorsque le narrateur de " Ode to a Nightingale " arrive à la fin de sa communion poétique avec l'oiseau, Keats emploie un tiret juste avant de poser la question « Do I wake or sleep? » Le narrateur semble retenir son souffle quelques secondes, comme pour préserver la vision, avant de mettre en doute son état de conscience et de revenir tout à fait au monde réel. La conclusion de « I stoop tip-toe » est construite sur un schéma similaire. Le locuteur, qui n'est pas encore tout à fait certain de son rôle, se demande : « Was there a Poet born? - but now no more - / My wand'ring spirit must no farther soar » (vers 241-42). Une fois encore, le narrateur se pose une question, retient son souffle, comme pour conserver l'inspiration en lui, puis expire, suite à quoi l'expérience poétique se termine tout à fait.

Enfin, la perte d'inspiration est souvent associée à l'idée d'étouffement et d'agitation psychique. Lorsqu'Apollon et son chariot disparaissent de la vision du narrateur de « Sleep and Poetry », il se sent oppressé par une impression de réalité. Son agitation est comparée à un flot d'eau boueuse (vers 15559). La perte d'inspiration est donc fortement liée à la sensation de noyade. Il est également intéressant de constater que Keats, malade de la tuberculose, cesse peu à peu d'écrire. L'amoindrissement de sa capacité respiratoire semble avoir influé sur sa perte d'inspiration et sur sa capacité à composer.

$7 \quad$ Pour plus de détail sur l'utilisation des tirets dans l'œuvre de Keats, on pourra se référer à l'ouvrage de Sylvie Crinquand, Lettres et poèmes de John Keats : portrait de l'artiste, qui y consacre un chapitre entier. 
Ainsi, les distinctions entre les sens et leurs fonctions propres disparaissent. Keats redéfinit en fait l'expérience sensorielle en l'intensifiant et en lui donnant un aspect de toile. ${ }^{8}$ Le contact chez Keats se présente en effet très souvent sous forme d'entrelacs, de guirlande ou de tressage. Une recherche dans l'ensemble des poèmes sur la base de données Literature Online 9 indique que les termes dont la racine est « wreath- » apparaissent à trente-six reprises dans l'œuvre et que des verbes ou noms portant le préfixe « inter-» reviennent vingt-trois fois au moins. Les verbes que précède le préfixe accentuent d'ailleurs cette idée. Dans Endymion, on retrouve « interknit» au vers 813 du premier livre et au vers 382 du troisième, et les verbes « intertwin'd », « interlace » et « interbreath'd » aux vers 412, 614 et 666 du livre deux respectivement.

Tous impliquent l'enveloppement, l'enchevêtrement et le contact. L'expérience fait s'entrelacer les sens, les sensations et les perceptions si bien qu'il devient presque impossible de les considérer un à un. Il apparaît alors nécessaire pour Keats de trouver un nouveau moyen d'exprimer l'expérience sensorielle, ce qu'il fait en se servant de la synesthésie comme d'un hypersens, un tressage de sensations.

Utiliser l'idée d'entrelacs pour définir la synesthésie keatsienne présente un avantage considérable. En effet, à travers l'image de la tresse, on associe plusieurs éléments en un tout cohérent dont chaque composant demeure pourtant identifiable, comme une symphonie assemble les sons de manière à créer une mélodie euphonique sans que les instruments ne perdent leur individualité. L'image de la tresse ou de la guirlande permet en outre d'accentuer l'aspect tactile qui participe de la singularité de l'expérience sensorielle keatsienne.

La synesthésie devient alors un sens issu du tressage des autres sens. L'œuvre de Keats constitue une synthèse de son expérience poétique qui elle-même unit réflexion, émotion et impressions sensorielles en un tout inextricable. Utiliser la synesthésie permet au poète d'intégrer les sensations physiques à son œuvre et de dépasser les limites de la perception pour arriver à un plus grand sentiment de complétude.

Le meilleur exemple de cette synthèse est le « bower » ou boudoir végétal keatsien. Lieu-clef de la création poétique, il est également l'espace où l'harmonie sensorielle est la plus apparente. On peut reprendre l'introduction du premier boudoir de « I stood tip-toe upon a little hill» pour exemple :

8 Pour une autre étude du motif de la toile dans l'œuvre de Keats, on pourra aussi voir : Sylvie Crinquand. « Une poétique du tissage : La Chute d'Hypérion, de John Keats (1821) ». Françoise Bort; Valérie Morisson. Texte, texture, textile. Variations sur le tissage dans la musique, les arts plastiques et la littérature, EUD, pp.57-66, 2013.

9 http://lion.chadwyck.co.uk.proxy-scd.u-bourgogne.fr/ 
And many pleasures to my vision started;

So I straightway began to pluck a posey

Of luxuries bright, milky, soft, and rosy.

A bush of May-flowers with the bees about them;

Ah, sure no tasteful nook would be without them;

And let a lush laburnum oversweep them,

And let long grass grow round the roots to keep them

Moist, cool, and green; and shade the violets,

That they may bind the moss in leafy nets. (vers 26-34)

Le plaisir induit par l'observation du paysage stimule l'imagination du poète qui commence alors sa description d'un creux de verdure de nature synesthésique. La juxtaposition des adjectifs « bright », « milky », « soft » et « rosy », qui appartiennent à quatre domaines sensoriels différents, la vue, le goût, le toucher et l'odorat, implique en effet l'harmonie et la simultanéité de perceptions distinctes. Synesthésie et création poétique se trouvent par conséquent liées. En outre, l'utilisation des adjectifs « milky » et « rosy » est synesthésique par essence car " milky » évoque autant la couleur que le goût du lait au lecteur tandis que « rosy » fait référence à la couleur, mais aussi au parfum des roses. Le poète nous emporte donc vers une réalité où les barrières entre les sens ont disparu et que seule la synesthésie permet de décrire.

En outre, les vers suivants reprennent l'image du tressage, image qui s'applique également aux sensations évoquées. Ainsi, la description du boudoir végétal en appelle à tous les sens humains : le toucher avec « moist», « cool », « bind » ou « oversweep », le goût avec l'adjectif « tasteful», l'ouïe à travers l'évocation des abeilles, la vue avec les couleurs et les jeux de lumière et l'odorat avec les références aux violettes et à l'herbe. Les sensations s'enlacent à l'image de la végétation qui forme des toiles (« nets ») et de l'herbe qui s'enroule autour des racines pour les aider à conserver leur fraîcheur et leur couleur. De plus, la synesthésie se poursuit sur plusieurs vers de sorte que le lecteur est contraint de ressentir, d'entendre et de voir en même temps comme si ses sens étaient tressés les uns aux autres. Le boudoir végétal keatsien est donc bien un espace de perception synesthésique, tant pour le poète que pour son lectorat.

John Barnard ${ }^{10}$ vient corroborer cette affirmation par une remarque intéressante sur l'épithète « lush » qui qualifie le cytise. Il explique en effet que l'adjectif fait appel aux sens du goût et du toucher,

10 Barnard, John. “Keats's Tactile Vision: 'Ode To Psyche' and the Early Poetry.” Keats-Shelley Review 33 (1982): 1-24. 
mais que Keats l'employait également pour désigner des couleurs vives (4). Ainsi, le schéma de toile qui se dessine dans la description se retrouve aussi dans les mots. Barnard utilise d'ailleurs la notion d'hyperesthésie dans son analyse des vers 9 à 12 du sonnet « Time's sea hath been five years » (6). Il me semble que l'emploi même de ce terme appuie l'idée que la synesthésie keatsienne se présente comme un « hypersens », un tout sensible au moyen duquel l'expérience sensorielle est redéfinie pour englober l'ensemble des domaines sensitifs.

Toutefois, les boudoirs végétaux ne sont pas les seuls exemples de synesthésie dans l'œuvre de Keats. Son emploi est étendu à de nombreux aspects de la poésie keatsienne. La présence de la femme aimée, par exemple, ne peut être décrite par les simples sens humains. C'est pourquoi Keats emploie la synesthésie dans la plupart des poèmes amoureux ou des romances.

Ainsi, lorsque Porphyro désire voir Madeline dans The Eve of St. Agnes, la vue ne lui suffit pas. En présence de sa bien-aimée, le jeune homme ne pourrait se contenter de la regarder ; il devrait au contraire ressentir toutes les sensations à la fois : lui parler, la toucher, l'embrasser, et même sentir son souffle. C'est pourquoi lors de leur union charnelle, Keats décrit l'acte d'amour comme le mélange subtil des odeurs et des couleurs de la rose et de la violette. L'acte sexuel devient ainsi synesthésique par essence. De la même façon, on peut citer la quatrième strophe de «Lines to Fanny »:

Save it for me, sweet love! though music breathe

Voluptuous visions into the warm air,

Though swimming through the dance's dangerous wreath:

Be like an April day,

Smiling and cold and gay,

A temperate lily, temperate as fair;

Then, Heaven! there will be

A warmer June for me. (vers 25-32)

Le poète pense à celle qu'il aime et retire de son souvenir l'inspiration nécessaire pour écrire le poème. Toutefois, cette inspiration est de nature clairement synesthésique : la musique respire des visions de volupté et de délectation qui suggèrent aussitôt le toucher et le goût. De plus, ces visions flottent dans un air chaud, et pourtant comparable à l'eau, si bien que la respiration et le toucher ne peuvent plus être distingués. On retrouve enfin le schéma d'entrelacs dans le terme « wreath ».

Quant à la femme aimée, elle évoque la synesthésie - en sa présence, le poète se figure un jour d'avril dont la température est encore fraîche, mais qui exhibe cependant des couleurs tièdes, le parfum 
d'une fleur de lys et la chaleur du mois de juin avenir. Les sensations sont donc bien combinées en un sens supérieur.

Pour finir, le lien entre synesthésie et jouissance dépasse la poésie pour s'exprimer dans la correspondance. Dans la lettre du 22 septembre 1819 de Keats à son ami C. W. Dilke, on peut lire :

Talking of Pleasure, this moment I was writing with one hand, and with the other holding to my Mouth a Nectarine - good god how fine - It went down soft pulpy, slushy, oozy - all its delicious embonpoint melted down my throat like a large beatified Strawberry. I shall certainly breed. (281)

Sylvie Crinquand explique que dans l'extrait, le plaisir provient plus encore de la sensation de la nectarine contre le palais que du goût du fruit (159). ${ }^{11}$ Or, si le plaisir du poète est issu à la fois du goût du fruit et du toucher palatal, il naît également de l'odeur alléchante de la nectarine et de son allure ronde, béate, comparable à celle d'une fraise. Une fois encore, la synesthésie associe tous les sens, ou presque, en les tressant de façon à rendre les sensations inextricables. En outre, comme la synesthésie du boudoir végétal, cette harmonie sensorielle est intimement liée à la création poétique. L'acte d'écriture et la description minutieuse des sensations sont des actions simultanées qui, comme Sylvie Crinquand le remarque à nouveau, vont jusqu'à évoquer la jouissance sexuelle et l'enfantement (159). Or, la jouissance et l'acte d'amour sont synesthésiques en ce qu'ils constituent l'enlacement de deux corps et de deux esprits.

Ainsi, synesthésie et création poétique, liées par les mots, apparaissent interdépendantes. En effet, on a déjà remarqué que les touchers keatsiens se manifestent par l'entrelacs, mais constituent également une forme de l'imagination et une partie de la création poétique. De la même façon, la synesthésie, tressage des sens destiné à n'en former qu'un, ne peut être dissociée de la création poétique. Elle devient un hypersens qui permet au poète de percevoir et de comprendre toute l'étendue du spectre des sensations conscientes. La synesthésie participe ainsi du désir keatsien de complétude. De plus, comme les autres sens, elle établit un lien étroit entre perception et création : l'état d'hypersensibilité dans lequel se trouve le poète lui donne la faculté de découvrir un monde de beauté qu'il peut ensuite rendre perceptible à son lecteur.

11 Crinquand, Sylvie. Lettres et poèmes de John Keats : portrait de l'artiste. Interlangues littératures, Dijon : Editions universitaires de Dijon; Toulouse : Presses universitaires du Mirail, 2000. 
Ce développement ne signifie pas néanmoins que le transfert d'un sens vers un autre soit totalement absent de l'œuvre de Keats. Il est en général la conséquence de l'insensibilisation du sens source. Ainsi, dans « Ode to a Nightingale », le locuteur explique :

I cannot see what flowers are at my feet,

Nor what soft incense hangs upon the boughs,

But, in embalmèd darkness, guess each sweet

Wherewith the seasonable month endows

The grass, the thicket, and the fruit-tree wild;

White hawthorn, and the pastoral eglantine;

Fast-fading violets cover'd up in leaves;

The coming musk-rose, full of dewy wine,

The murmurous haunt of flies on summer eves. (41-50)

Helen Vendler, dans son analyse de l'ode, soutient que le locuteur perd peu à peu tous ses sens, qui se trouvent finalement limités à l'ouïe. Son état d'insensibilité est presque complet et lui permet d'oublier les souffrances comme les joies humaines (100). ${ }^{12}$

Quoique l'ode puisse effectivement laisser penser que l'expérience sensorielle est peu à peu réduite à l'audition du locuteur, il me semble toutefois qu'il s'agit d'un transfert sensoriel provoqué par cette insensibilisation de l'oeil. En effet, si le locuteur ne peut voir les fleurs à ses pieds ou l'encens qui flotte dans les airs, il est toutefois conscient de leur odeur, comme l'indique l'expression « embalmèd darkness ». Ses facultés visuelles sont transférées à son sens de l'odorat, par lequel il peut deviner ("guess ») le nom et les couleurs des fleurs - bien qu'il ne puisse les voir, il reconnaît les églantines, l'aubépine, les violettes et les roses musquées à leur parfum. Finalement, c'est l'imagination olfactive qui permet au poète de retrouver les couleurs de ces fleurs comme s'il pouvait vraiment les voir. Son sens de la vue étant insensibilisé, le locuteur pallie la perte de ce sens grâce à un transfert de ses attributs sur des sens demeurés intacts.

On trouve un exemple similaire de transfert dans The Fall of Hyperion :

Methought I stood where trees of every clime,

Palm, myrtle, oak, and sycamore, and beech,

With platane and spice blossoms, made a screen,

In neighbourhood of fountains (by the noise

Soft-showering in mine ears), and (by the touch

Of scent) not far from roses. (vers 19-24)

12 Vendler, Helen. The Odes of John Keats. Cambridge: The Belknap Press of Harvard University Press, 1983. 
Les arbres de tous climats forment un écran («screen ») qui empêche le poète de voir le paysage au-delà de la forêt - il est par conséquent comme aveuglé. Cependant, si son sens de la vue est défaillant, par un transfert sur les autres sens, il peut recouvrer cette vue perdue. En effet, les fontaines apparaissent devant ses yeux lorsqu'il les entend ; on remarquera d'ailleurs que l'emploi de l'expression " noise / soft-showering in mine ears » n'indique pas seulement que le poète entend les fontaines dont il se figure l'apparence. On a l'impression qu'il voit l'eau qui coule doucement autant qu'il en perçoit le son. En outre, lorsqu'il perçoit la senteur des roses, le locuteur semble se les représenter. Ainsi, la vision devient olfactive, tactile, et auditive, si bien que même si elle a disparu, elle demeure active par l'action des autres sens. Lorsque l'un des sens est insensibilisé, ses attributs sont transférés sur les sens encore conscients ou fonctionnels. Ainsi, la disparition partielle de sensations devient presque vecteur de la synesthésie.

Aussi la synesthésie keatsienne prend-elle deux formes : hypersens, elle redéfinit l'expérience sensorielle en masquant les barrières entre les sens et en permettant un tressage sensitif ; transfert, elle permet de pallier l'insensibilisation d'un des organes sensoriels et fait ainsi le pont entre le monde sensible et ce qui ne peut être ressenti.

La richesse et la complexité de la synesthésie keatsienne en font par conséquent toute la singularité et influencent les caractéristiques du poète idéal keatsien à qui elle permet de ressentir les souffrances et les joies du monde comme si elles étaient siennes. Lorsqu'il écrit à Benjamin Bailey « $\mathrm{O}$ for a Life of Sensations rather than of Thoughts! » dans sa célèbre lettre du 22 novembre 1817, Keats insiste sur l'importance de l'expérience sensorielle. L'homme ne peut connaître le monde et découvrir la vérité qu'à travers ses sensations et son imagination. De plus, si l'on doit préférer la sensation à la raison pure, c'est bien parce qu'elle est également porteuse d'un sens philosophique sans lequel toute création poétique serait inenvisageable. Si l'on reprend le dialogue entre le locuteur de The Fall of Hyperion et Moneta, on constate en effet que le poète keatsien doit être à la fois capable de sympathie et d'empathie, au sens moderne de ces deux termes :

Majestic shadow tell: sure not all

Those melodies sung into the world's ear

Are useless: sure a poet is a sage;

A humanist, physician to all men (vers 187-90).

$[\ldots]$

The poet and the dreamer are distinct,

Diverse, sheer opposite, antipodes.

The one pours out a balm upon the world,

The other vexes it. (vers 199-202) 
Le poète est un sage, un humaniste et un médecin. Or, le sage est celui qui connaît le monde ; il symbolise la maturité. De la même façon, l'humaniste comprend les malheurs et les joies humaines. Le poète est donc un être doué d'empathie, capable de se mettre à la place des autres pour les comprendre sans perdre son identité. Par ailleurs, le poète est un médecin qui ressent et doit apaiser les souffrances de la terre, besoin qui naît de la sympathie. Il semble donc que chez le poète keatsien, les deux facultés cohabitent. La sympathie pour l'homme pousse le poète keatsien à devenir son guérisseur et l'empathie lui permet de comprendre et d'analyser la source de la douleur pour mieux la soigner.

Ainsi, lorsque le locuteur de « Ode to a Nightingale » décrit les souffrances humaines, on partage sa sympathie pour l'humanité :

Here, where men sit and hear each other groan;

Where palsy shakes a few, sad, last grey hairs,

Where youth grows pale, and spectre-thin, and dies;

Where but to think is to be full of sorrow

And leaden-eyed despairs;

Where Beauty cannot keep her lustrous eyes,

Or new Love pine at them beyond to-morrow. (vers 24-30)

La synesthésie, définie comme un entrelacs de sensations, est présente dans l'extrait cité. On retrouve le sens de l'ouïe dans l'expression « where men sit and hear each other groan ». Le toucher vient ensuite se lier à ce sens dans la proposition « palsy shakes a few, sad, last grey hairs ». Les sensations restent liées jusqu'à la fin de la strophe, avec l'adjectif composé « leaden-eyed » qui en appelle au toucher et à la vue. En effet, le plomb est un métal lourd, mais évoque également une couleur d'yeux. De la même façon, la jeunesse est à la fois pâle et maigre. Or, on perçoit la maigreur par l'action de deux sens : le toucher et la vue. Contrairement à la synesthésie luxuriante des boudoirs végétaux, l'harmonie des sensations est ici négative et intimement liée à la douleur, comme l'indiquent le verbe " dies » ou la proposition "Where Beauty cannot keep her lustrous eyes, / Or new Love pine at them beyond to-morrow ».

Les émotions et les sentiments se mêlent ensuite à l'expérience sensorielle pour former un tout. On rejoint alors l'une des définitions de la synesthésie proposées par The Oxford English Dictionary : la synesthésie permet l'adéquation entre les sensations et les sentiments si bien qu'elle devient une étape clé dans le développement de la sympathie.

Ainsi, ce ne sont pas les hommes qui ont un regard de plomb (« leaden-eyed»), mais le désespoir. Le jeune homme et la jeune femme qu'on imagine les yeux brillants sont remplacés par l'amour et la 
beauté. Les émotions et les sentiments prennent ainsi des attributs humains qui entraînent la sympathie du lecteur. Ce dernier, par le jeu d'associations lexicales du poème, est conduit à mettre en relation le désespoir et la douleur avec l'humanité.

Cependant, le poème ne s'achève pas sur une note aussi sombre. Après avoir éveillé la sympathie du lecteur, le locuteur lui offre, dans la strophe suivante, la possibilité de soulager son esprit en se laissant transporter par la poésie. L'ode crée en fait un contraste entre la beauté (ou le bonheur) et les souffrances humaines grâce auquel le locuteur partage son expérience sympathique avec le lecteur en lui dévoilant les malheurs de l'humanité puis en signifiant son besoin de les apaiser. ${ }^{13}$

La synesthésie permet ainsi au poète de partager ses réactions sympathiques et empathiques avec le lecteur. Ce nouveau sens le conduit également à des questionnements sur la nature de la douleur pour faire naître en lui le désir d'apaiser les souffrances humaines : pour Keats, le poète doit être, ou tout du moins, tenter d'être, un médecin de l'âme humaine. Le vocabulaire médical, allant des références aux poisons à la répétition de termes tels que « soothe », « balm », « heal » et « health » et leurs dérivés, occupe d'ailleurs une place importante dans les poèmes comme dans la correspondance.

Le poète-médecin cherche alors à procurer un soin par le plaisir des sens. Grâce à la synesthésie, il dévoile au lecteur un monde de beauté dans lequel l'expérience sensorielle est entière, et lui fait oublier, l'espace d'un instant, la douleur née de la perte et du passage implacable du temps.

13 Cet aspect est encore plus marqué dans The Fall of Hyperion. 


\section{Ouvrages Cités}

BARNARD, John. « Keats's Tactile Vision: 'Ode To Psyche' and the Early Poetry ». Keats-Shelley Review 33 (1982): 1-24.

CRINQUAND, Sylvie. Lettres et poèmes de John Keats : portrait de l'artiste. Interlangues littératures, Dijon : Editions universitaires de Dijon ; Toulouse : Presses universitaires du Mirail, 2000.

—. «Une poétique du tissage : La Chute d'Hypérion, de John Keats (1821)». in Bort Françoise, Morisson Valérie, eds. Texte, texture, textile. Variations sur le tissage dans la musique, les arts plastiques et la littérature. Dijon : Editions universitaires de Dijon, 2013: 57-66.

ERHARDT-SIEBOLD, Erika von. « Harmony of the Senses in English, German, and French Romanticism ». PMLA: Publications of the Modern Language Association of America, 47.2 (1932): 577-592.

FINCH, G.J. « Wordsworth, Keats and the 'language of the sense' ». Ariel: A Review of International English Literature Calgary, 11.2 (1980): 23-36.

GIGANTE, Denise. « Keats's Nausea ». Studies in Romanticism, 40.4 (2001): 481-510.

KEATS, John. Bright Star: The Complete Poems and Selected Letters of John Keats. London: Vintage Books, 2009.

—. John Keats: Selected Letters, Gittings, Robert, ed. Revised by Jon Mee. Oxford: Oxford University Press, 2002.

LAMBERT, Hervé-Pierre. « La synesthésie. Vues de l'intérieur ». Epistémocritique, Vol. VIII (2011). http://www. epistemocritique.org/spip.php?article210\# ftn48

SEGAL, Naomi. «'L'échange de deux fantaisies et le contact de deux épidermes': Skin and Desire », in Syrotinski Michael, and Maclachlan Ian, eds. Sensual Reading: New Approaches to Reading in its Relations of the Senses. London: Associated University Presses, 2001: 17-38.

SHEN, Yeshayahu, and Cohen Michal. " How Come Silence is Sweet but Sweetness is not Silent: A Cognitive Account of Directionality in Poetic Synaesthesia ». Language And Literature: Journal of the Poetics and Linguistics Association, 7.2 (1998): 107-121.

TSUR, Reuven. «Issues in Literary Synaesthesia », Style, 41.1 (Spring 2007): 30-52.

ULLMANN, Stephen de. « Romanticism and Synaesthesia: a Comparative Study of Sense Transfer in Keats and Byron ». PMLA: Publications of the Modern Language Association of America, 60.3 (1945): 811-827.

VENDLER, Helen. The Odes of John Keats. Cambridge: The Belknap Press of Harvard University Press, 1983.

WORDSWORTH, William. «Tintern Abbey ». William Wordsworth: The Major Works (including The Prelude). Stephen Gill, ed. Oxford: Oxford University Press (Oxford World's Classics), 2008.

WALDOFF, Leon. Keats and the Silent Work of Imagination. Urbana: University of Illinois Press, 1985. 